\title{
INDUCTANCE CALCULATIONS OF PRINTED WINDINGS OF POWER TRANSFORMERS USING PARTIAL INDUCTANCE METHOD
}

\author{
Wing C. Ho \\ M. H. Pong \\ Department of Electrical and Electronic Engineering \\ The University of Hong Kong
}

\begin{abstract}
$A B S T R A C T$ - It is a common occurrence that hand-wound or machine-wound transformers have the problem of wide variation in parameter values inductance and leakage inductance, etc. - in a lot of sample. These are partly due to the tolerance of ferrite core but more importantly it is the result of inconsistent spacings between loosely packed windings as inductance and leakage are geometric shape dependent. The method of printed windings of transformer -windings are printed on a printed circuit board (PCB) and all windings are embedded in a stack of these PCBs - is a promising way in controlling the windings' shape and air spacing in a fixed pattern. While it eliminates the parameter variations in a great deal. However, traditional analytical methods of inductance calculations are based on a lot of assumptions. The complicated geometric constructions of transformer also hinder the accuracy of calculations. The partial inductance method gives an easy and accurate approach to calculate loop inductance by the generalised partial inductance, which is derived from dividing the whole loop into finite length of elements. Self and mutual inductance are then the summation of inductance contributed from individual elements. This is useful in predicting the leakage inductance which has been the most undesirable parameter in PWM converter. And this method can also be applied to transformers in resonant converters in which inductances have to be accurately controlled.
\end{abstract}

\section{INTRODUCTION}

No windings or transformers can be built identically by hand-wound or machine-wound methods. Inductance and leakage inductances can vary widely between transformers because of the inconsistent spacings between windings of traditional transformer, even though they are under the same design procedure. However, with the windings printed on a PCB in a desirable fixed pattern, and then stacked up several layers of PCB for having multiple turns, it is possible to have a consistent value of inductance and leakage inductances between transformers. The next step of design is to manipulate inductance so as to reduce or optimise the leakage inductance in design purpose. The calculation of inductance, especially the leakage inductance of power transformer, has been the major problem faced by many switched-mode power supply (SMPS) designer as it is hard to have an accurate value by simple analytical calculation. Designer can only make an approximation by engineering sense or experience when facing some complicated structure or loosely packed windings of transformer. This may result in high voltage spikes as unknown or uncontrollable leakage inductance present during switching action in a converter. The partial inductance method [1] is firstly introduced for calculating multiloop inductances in a complex integrated circuit environment. However, the procedures proposed in [1] cannot directly handle the partial self inductance. It substitutes a long mathematical expression from Table for partial self inductance, and hence, makes the whole procedure become clumsy. In this paper, Section V handles the above problem in a nice manner so that the whole procedure can be performed efficiently.

\section{PRINTED WINDINGS OF TRANSFORMER}

The idea of printed windings of transformer can be easily understood by looking at Fig. 1. A few turns of windings are being printed on the top of a PCB. The thickness, width and number of turns are well defined by the design procedure of power transformer. With the interconnection between layers, one can see that the windings of layers are moving around in the same direction either clockwise or anti-clockwise. The secondary windings are put at the edge of PCB, encircling the primary windings. The whole stack of layers, in which upper and lower layers are joint by via holes, form a complete isolated stack of windings. The following section introduces the theories by which the inductances are calculated.

\section{SELF AND MUTUAL INDUCTANCE OF COUPLED CIRCUIT}

\section{(A) ANALYTICAL APPROACH}

For a coupled circuit in a linear system, the self and mutual inductance can be firstly calculated from the concept of energy stored in the magnetic fields [2], and then expressed in terms of magnetic vector 


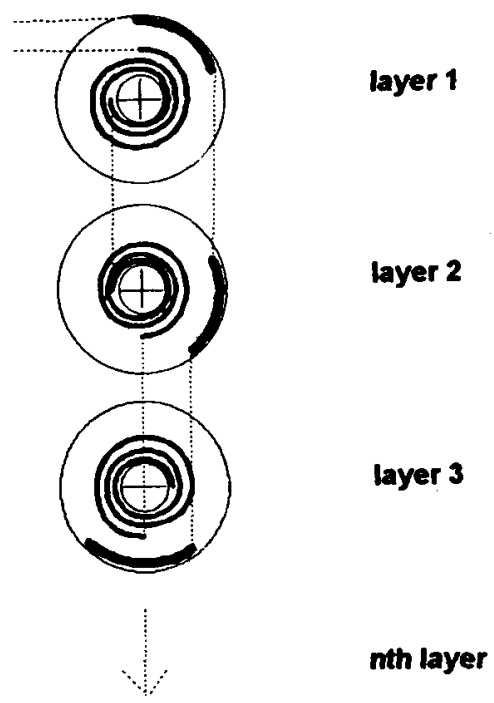

Fig. 1 Section layers of printed transformer with printed windings on PCBs

potential $\mathbf{A}$ and current density $\mathbf{J}$. The subscript 1 and 2 indicate the corresponding circuit loops.

$$
\begin{array}{r}
U_{m}=\frac{1}{2} \int_{v 1} \mathbf{A}_{1} \bullet \mathbf{J}_{1} d v+\frac{1}{2} \int_{v_{2}} \mathbf{A}_{2} \bullet \mathbf{J}_{2} d v \\
+\frac{1}{2} \int_{v_{2}} \mathbf{A}_{1} \bullet \mathbf{J}_{2} d v+\frac{1}{2} \int_{v 1} \mathbf{A}_{2} \bullet \mathbf{J}_{1} d v \\
\text { [Joules] }
\end{array}
$$

Using the definition of energy stored in an inductor, $U=\frac{1}{2} L I^{2}$ joules, one can reveal the self and mutual inductance in the following manner.

$$
\begin{aligned}
& L_{1}=\frac{1}{I_{1}^{2}} \int_{v 1} \mathbf{A}_{1} \bullet \mathbf{J}_{1} d v \\
& \text { self inductance of loop } 1 \text { circuitry } \\
& L_{2}=\frac{1}{I_{2}^{2}} \int_{v 2} \mathbf{A}_{2} \bullet \mathbf{J}_{2} d v \\
& \text { self inductance of loop } 2 \text { circuitry } \\
& M_{21}=\frac{1}{I_{1} I_{2}} \int_{v 2} \mathbf{A}_{1} \bullet \mathbf{J}_{2} d v
\end{aligned}
$$

mutual inductance of loop 2 due to loop 1 circuitry

$$
M_{12}=\frac{1}{I_{1} I_{2}} \int_{v 1} \mathbf{A}_{2} \bullet \mathbf{J}_{1} d v
$$

mutual inductance of loop 1 due to loop 2 circuitry

\section{(B) FULL EXPRESSION OF MUTUAL INDUCTANCE}

It is true for our printed transformers that the thickness of windings can be well controlled. Usually the copper traces on the PCB are thin, around $1 \mathrm{~mm}$ in thickness. With the converter operating below 482
$\mathrm{MHz}$, the effect of current redistribution on copper area should not occur as the thickness of windings is well below the effective skin depth. Therefore, one can make the assumptions that :

(a) current density in the source points of circuitry is constant or evenly distributed

(b) skin effect and proximity effect is nearly negligible

With the amount of current I flowing through a surface, it can be formulated that

$$
I=\int_{S} J d s \quad[\mathrm{~A}]
$$

The definition of magnetic vector potential $\mathbf{A}$ is

$$
\mathbf{A}=\frac{\mu}{4 \pi} \int_{v} \frac{\mathbf{J}}{r} d v
$$

By substituting the expression (6) and (7) into (4) or (5), the mutual inductance then becomes

$$
M_{21}=\oint_{l 2} \oint_{l 1} \frac{\mu}{4 \pi} \frac{1}{S_{1} S_{2}} \int_{S 1} \int_{S 2} \frac{d l_{1} d l_{2}}{r_{12}} d S_{1} d S_{2}
$$

The other three expressions of inductance can also be derived from the same procedure.

\section{DERIVATION OF PARTIAL INDUCTANCE}

The concept of partial inductance comes from the point that the parameter $d \mathbf{l}_{k} \bullet d_{m}$ can actually be two finite length of segments spatially separated by a distance $r_{k m}$ and oriented in corresponding current flow direction. Fig. 2 illustrates part of elements in an arbitrary length of loop. The associated flux linking the two elements are bounded by the area in which 
the edges consist of the related elements and extended portion if necessary and two perpendicular lines joining ends to ends of elements. By the use of $\mathbf{B}=\vec{\nabla} \times \mathbf{A}$, the associated flux is then to be $\oint_{l} \mathbf{A d a}$.

It is realised that when taking closed loop integration of the bounded area, the flux contributed from paths that are perpendicular to each other become vanished. The remaining contributions come from those of paths $d_{k}$ and $d_{m}$.Therefore, the expression of the associated flux is

$$
\Rightarrow \begin{aligned}
\Phi_{(\text {partial })} & =\oint_{l} \mathbf{A d} \\
& =J_{k} J_{m} \int_{\Delta k} \int_{\Delta \mathrm{m}} \int_{S k} \int_{S m} \frac{\mu}{4 \pi} \frac{d S_{k} d S_{m}}{r_{k m}} d l_{k} d d_{m}
\end{aligned}
$$

And the partial inductance with permeability $\mu$ in medium is

$$
\begin{gathered}
L(\mu)_{p k m}=\frac{\Phi_{\text {(portia) }}}{I_{k} I_{m}} \\
\quad=\frac{\mu}{4 \pi} \frac{1}{S_{k} S_{m}} \int_{S k} \int_{S m} \int_{\Delta k k} \int_{\Delta m} \frac{1}{r_{b m}} d l_{k} d 1_{m} d S_{k} d S_{m}
\end{gathered}
$$

For simplicity of calculation, the area terms can be dropped out as long as the concerned areas are in uniform and consistent shape. And then,

$$
L(\mu)_{p k m}=\frac{\mu}{4 \pi} \int_{\Delta k k} \int_{\Delta l m} \frac{d d_{k} d d_{m}}{r_{k m}}
$$

And the partial inductance in free space is

$$
L\left(\mu_{o}\right)=\frac{\mu_{o}}{4 \pi} \int_{\Delta l k} \int_{\Delta l m} \frac{d_{k} d d_{m}}{r_{k m}}
$$

Replacing the expression (8) with the partial inductance, we finally get the mutual inductance of loop $\mathrm{i}$ and loop $\mathrm{j}$ as

$$
\begin{aligned}
M(\mu)_{i j}= & \oint_{l i} \oint_{l j} L(\mu)_{p k m} \\
= & \sum_{l i} \sum_{l j} L(\mu)_{p k m} \quad[\mathrm{H}] \\
& \text { in medium with permeability } \mu
\end{aligned}
$$

$$
\begin{aligned}
M\left(\mu_{o}\right)= & \oint_{l i} \oint_{l j} L\left(\mu_{o}\right)_{p k m} \\
= & \sum_{l i} \Sigma_{l j} L\left(\mu_{o}\right)_{p k m} \quad[\mathrm{H}] \\
& \text { in free space }
\end{aligned}
$$

By the same token, when the index $j$ changes to $i$, the value becomes self inductance of a loop

$$
L(\mu)_{i i}=\Sigma_{l i} \Sigma_{l i} L(\mu)_{p k m} \quad[\mathrm{H}]
$$

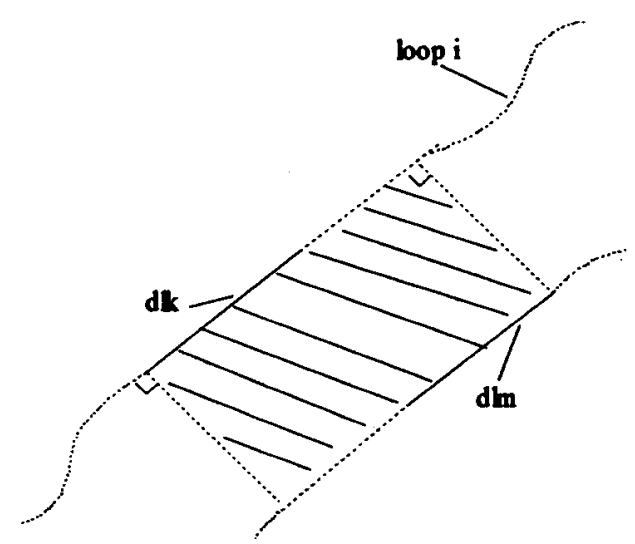

Fig. 2 Diagram of partial flux due to partial segments dlk and dlm

\section{THE PROBLEM OF SINGULARITY IN PARTIAL SELF INDUCTANCE}

It is noted that there may be a singular point present in the partial self inductance $L_{k k}$ as $\frac{1}{r_{k k}}$ goes to infinitive when $r_{k t}$ goes to zero. It means that there may be a problem of calculating two finite elements positioning coincidentally. However, when we consider the definition of magnetic vector potential A (7) again, we discover that the volume integral goes to zero when $r$ goes to zero. It is because for the current density $\mathbf{J}$, providing that it is a continuous function over the surface of a sphere, we can have a maximum value of $J_{\max }$ such that

$$
\begin{gathered}
\int_{v} \frac{J}{r} d v<\int_{0}^{r} \int_{0}^{4 \pi r^{2}} \frac{J_{\max }}{r} d s d r \\
\Rightarrow \int_{v} \frac{J}{r} d v<2 \pi r^{2} J_{\max } \\
\text { goes to zero as } \mathrm{r} \rightarrow 0
\end{gathered}
$$

It implies that there is no inductance value for a single point element. Therefore, it is possible to exclude the calculation of the partial self inductance $L_{k k}$, with a finite length of $\Delta l$, still keeping an accurate calculation as high as possible by reducing $\Delta l$, instead of using expression from mathematical handbook. It is a compromise to be made between accuracy and computing time.

\section{COUPLING COEFFICIENT, $K$}

\section{(A) FREE SPACE}

The coupling coefficient $K$ has been well defined by

$$
M=K \sqrt{L_{1} L_{2}}
$$




$$
\Rightarrow K^{2}=\frac{M^{2}}{L_{1} L_{2}}=\frac{M_{12} M_{21}}{L_{22} L_{11}}
$$

Representing $K^{2}$ in terms of partial inductance

$$
K^{2}=\frac{\left(\Sigma_{l 1} \Sigma_{l 2} L\left(\ddot{\mu}_{o}\right)_{p k m}\right)^{2}}{\left(\Sigma_{l 1} \Sigma_{l 1} L\left(\mu_{o}\right)_{p k m}\right)\left(\Sigma_{l 2} \Sigma_{l 2} L\left(\mu_{o}\right)_{p k m}\right)}
$$

The coupling coefficient can then be calculated numerically.

\section{(B) MEDIUM WITH PERMEABILITY $\mu_{o}$ AND $\mu$}

In practical situation, the windings' coupling effect can be increased by raising up the permeability of magnetic path, for instance, the insertion of a ferrite core in a transformer. However, windings are still not $100 \%$ coupled as mostly due to loosely packaging of windings. The air spacing between windings and ferrite core makes the integral path more complicated than that of in single medium. Fig. 3 shows the cross section layout of a typical one-turn printed transformer, with ferrite core having permeability $\mu$, in between coupled loops. Air spacing is represented by the region with $\mu_{o}$. The closed loop integral is consisting of outer path $C_{11}$, inner path $C_{21}$ and innermost path $C_{31}$. Where the index 1 appears in the subscript of $C_{31}$ indicates the corresponding loop 1 , vice versa. With the help of equations (13) and (14), the self and mutual inductance of the coupled circuit can be expressed as

(a) Self inductance of loop 1

$$
\begin{aligned}
& L_{11}=\oint_{C 11-C 12+C 31}\left[\oint_{C 11-C 21} L\left(\mu_{o}\right)_{p k m}\right. \\
& \left.\oint_{C 31} L(\mu)_{p k m}\right] \\
= & {\left[\Sigma_{C 11} \Sigma_{C 11} L\left(\mu_{o}\right)_{p k m}-\Sigma_{C 21} \Sigma_{C 21} L\left(\mu_{o}\right)_{p k m}\right]^{2} } \\
& +\Sigma_{C 11} \Sigma_{C 31} L(\mu)_{p k m}-\Sigma_{C 21} \Sigma_{C 31} L(\mu)_{p k m} \\
& +\Sigma_{C 31} \Sigma_{C 31} L(\mu)_{p k m}
\end{aligned}
$$

(b) Mutual inductance of loop 2 due to current in loopl

$$
\begin{aligned}
& M_{21}=\oint_{C 12-C 22+C 32}\left[\oint_{C 11-C 21} L\left(\mu_{o}\right)_{p k m}\right. \\
& \left.+\oint_{C 31} L(\mu)_{p k m}\right] \\
= & \Sigma_{C 12} \Sigma_{C 11} L\left(\mu_{o}\right)_{p k m}-\Sigma_{C 22} \Sigma_{C 11} L\left(\mu_{o}\right)_{p k m} \\
& +\Sigma_{C 32} \Sigma_{C 11} L\left(\mu_{o}\right)_{p k m}-\Sigma_{C 12} \Sigma_{C 21} L\left(\mu_{o}\right)_{p k m} \\
& +\Sigma_{C 22} \Sigma_{C 21} L\left(\mu_{o}\right)_{p k m}-\Sigma_{C 32} \Sigma_{C 21} L\left(\mu_{o}\right)_{p k m} \\
& +\Sigma_{C 12} \Sigma_{C 31} L(\mu)_{p k m}-\Sigma_{C 22} \Sigma_{C 31} L(\mu)_{p k m} \\
& +\Sigma_{C 32} \Sigma_{31} L(\mu)_{p k m}
\end{aligned}
$$

The coupling coefficient of this kind of configuration is that given by equation (19).

And the leakage inductances of primary and secondary windings of a transformer, shown in Fig. 4, can then be formulated as

$$
L_{p_{-} \text {leakage }}=(1-K) L_{11}[\mathrm{H}]
$$

primary leakage inductance

$$
L_{s_{\text {_leakage }}}=(1-K) L_{22}[\mathrm{H}]
$$

secondary leakage inductance

\section{WORKED EXAMPLES}

The following table shows some of the primary works that have been done on some simple configurations of windings. The results obtained by partial inductance method and those by analytic method are tabulated and compared. Table also shows a very closely match-up of figures.

\section{CONCLUSION}

The method of printed windings of transformers is a promising way of controlling the consistency of inductance values of transformers. The inductance and leakage inductance are then under control by design. The theory and concept of partial inductance method have been introduced. The method is applicable to any configurations of windings and medium path as long as the coupled circuit is in the linear system. The self, mutual and leakage inductances can be well predicted by the method. This
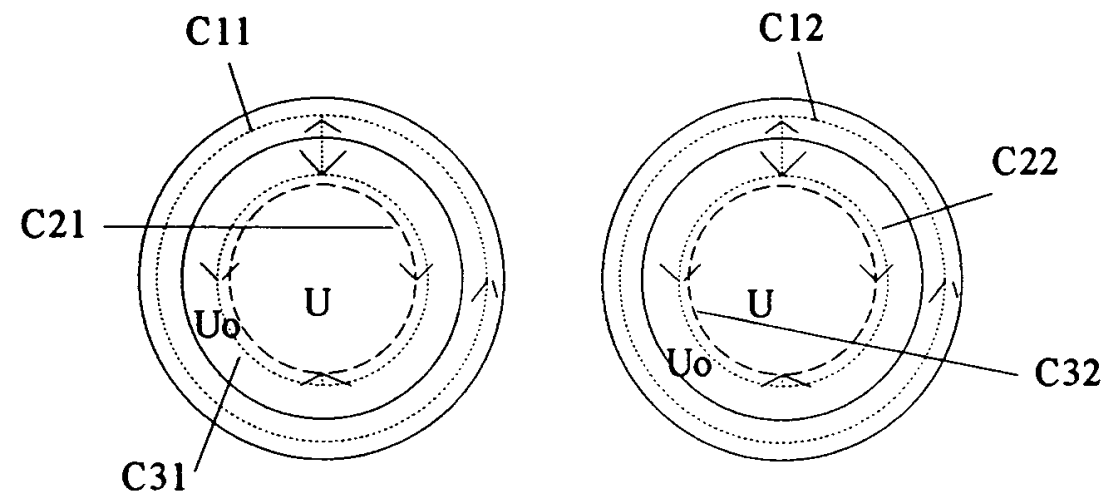

Fig. 3 Windings structure with permeability $u_{o}$ and $u$ in medium 
is useful and helpful in designing PWM or resonant converters as the leakage inductance can be more predictable and controllable.

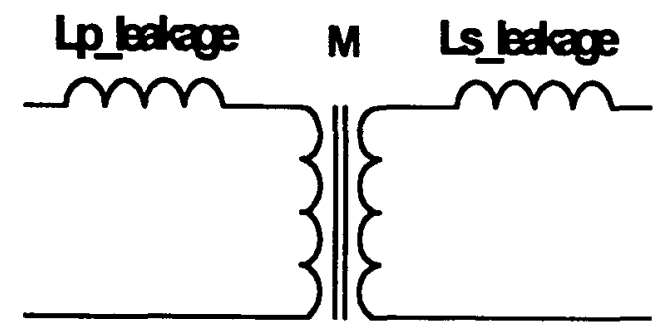

\section{REFERENCES}

[1] A. E. Ruehli, "Inductance Calculations in a Complex Integrated Circuit Environment", IBM J. Res.Develop., Sept. 1972

[2] Carl T. A. Johnk, "Engineering Electromagnetic Fields and Waves", John Wiley \& Son, Inc.

[3] A. E. Guile, "Electrical Power Systems", Pergamon Press, 1977

[4] W. P. King, S. Prasad, "Fundamental Electromagnetic Theory and Applications", Prentice Hall, 1986

[5] J. Frey, "Microwave Integrated Circuits", Artech House, 1975

Fig. 4 Transformer with primary and secondary leakage inductances

\begin{tabular}{|l|c|c|c|c||}
\hline & finite line $^{1}$ & single loop & double loop $^{3}$ & spiral $^{4}$ \\
\hline analytic & 0.41 & 0.26 & 1.29 & 1.63 \\
\hline partial inductance & 0.4 & 0.27 & 1.29 & 1.55 \\
\hline$\%$ differences & 2.4 & 3.8 & 0 & 4.9 \\
\hline 1 & See Ref. [3] & & \\
2 & See Ref. [2] & & & \\
3 & See Ref. [4] & & & \\
4 & See Ref. [5]
\end{tabular}

Table 1 Numerical and analytic inductance values of different configurations of winding, the above figures are in $\mu_{o}[\mathrm{H}]$ 